Results Five studies were included in the current analysis enrolling 223 women (191 with EC and 32 with CC) and 484 SLNs. The quality of the included studies was high. The number of the examined SLNs per patient ranged between one and five. The pooled sensitivity and specificity was 0.84 $(95 \%$ CI $0.64-0.94, \mathrm{I} 2=34.59 \%)$ and $0.95 \quad(95 \% 0.88-$ 0.98 , I2 $=87.58 \%$ ), respectively. The pooled LR+ and LRwas 17.07 and 0.17 , respectively. The pooled DOR was calculated 100.38 (95\% CI $34.21-294.52, \mathrm{I} 2=85.24 \%)$. The SROC curve yielded an AUC of 0.95 (95\% CI 0.93 - 0.97). Conclusion The current evidence suggests that the OSNA assay is a useful and accurate technique for the intra-operative detection of SLN metastasis in early-stage EC and CC. The combined analysis using SLNs and OSNA assay is seemingly an attractive approach to tailor individualised management. The impact of micro-metastasis and isolated tumour cells on the prognosis of women with apparent early-stage EC and CC remains debatable and should be addressed in future research. As this evidence is preliminary, cross-institutional collaboration is warranted.

Disclosures Professor SK declares personal fees for consulting from Roche and Astra-Zeneca, outside the submitted work. The remaining authors certify that no party has a direct interest in the results of the research and that no benefit will be conferred to us or any organisation with which we are associated.

\section{PROGNOSTIC VALUE OF PET-CT SCAN ON SURVIVAL OUTCOMES OF ADVANCED-STAGED OVARIAN CANCER PATIENTS TREATED WITH NEOADJUVANT CHEMOTHERAPY: A PROSPECTIVE STUDY}

${ }^{1}$ Stamatios Petousis, ${ }^{2}$ Anne-Laure Cazeau, ${ }^{2}$ Amandine Crombe, ${ }^{3}$ Chrysoula MargioulaSiarkou, ${ }^{2}$ Michel Kind, ${ }^{2}$ Sabrina Croce, ${ }^{2}$ Anne Floquet, ${ }^{2}$ Frederic Guyon. ${ }^{2}$ 2nd Department of Obstetrics and Gynaecology, Aristotle University of Thessaloniki; ${ }^{2}$ Institut Bergonie, Bordeaux, France; ${ }^{3}$ Institut Bergonie, Bordeaux, France; 2nd Department of Obstetrics and Gynaecology, Aristotle University of Thessaloniki, Greece

\subsection{6/ijgc-2020-ESGO.50}

Introduction/Background PET-CT is an imaging examination whose preoperative diagnostic value in advanced-staged ovarian cancer patients remains controversial. Main objective of this study was to answer whether performing early preoperative PET-CT scan in patients undergoing neoadjuvant chemotherapy may discriminate their response and prognosis.

Methodology A prospective observational study was performed between September 2014 and May 2016. There were exclusively included patients diagnosed with advanced-stage ovarian cancer considered as not eligible for primary debulking surgery according to laparoscopy Fagotti score. These patients were treated with four cycles of neoadjuvant chemotherapy with carbotaxole followed by interval debulking and three additional cycles of chemotherapy. PET-CT was performed between the initiation of chemotherapy (T0), first (T1) and fourth cycle of chemotherapy (T4). Follow-up outcomes of patients were also recorded. Primary outcomes were SUV (Standardized Uptake Value), MTV (Metabolic Tumor Volume) and TLG (Tumor Lesion Glycolysis) that were assessed by three different blind physicians each. Total and percentage modifications of these parameters within T0,T1 and T4 were compared between patients with and without recurrence and cancer-related death, while they were also correlated with OS and DFS in a Cox regression analysis.
Results $\tau$ here were 10 patients recruited for this study. All patients managed to have complete excision of the disease. SUVmax, MTV and TLG did not present significant interobserver variability within physicians. SUVmax was reduced at 45.9\% between $\mathrm{T} 0$ and $\mathrm{T} 1$ in patients with later cancerrelated death vs. only $8.0 \%$ in survivors $(\mathrm{P}=.05)$, while the relative mean decrease in absolute units was 6.5 vs 1.17 $(\mathrm{P}=.06)$. Similarly, TLG between $\mathrm{T} 0$ and $\mathrm{T} 1$ was reduced at $76.51 \%$ vs. $33.7 \%(\mathrm{P}=.04)$, while mean TLG decrease was 1663.8 vs 653.8 units respectively $(\mathrm{P}=.06)$. In contrary, patients not presenting recurrence were characterized by significantly higher TLG reduction between $\mathrm{T} 1$ and T4 $(95.0 \%$ for non-recurrence vs $69.1 \%$ for recurrence, $\mathrm{P}=.04$ ), while TLG mean reduction was 1088 vs. 211 units $(\mathrm{P}=.11)$. Furthermore, all mean values of PET-CT parameters presented a higher reduction between $\mathrm{T} 1$ and $\mathrm{T} 4$ in patients not presenting recurrence.

Conclusion PET-CT examination preoperatively in advancedstaged ovarian cancer patients may be prognostic. Further studies with larger sample size should be performed in order to assess the exact role of PET-CT scan on preoperative triage of advanced-stage ovarian cancer patients.

Disclosures Authors have nothing to disclose.

\section{Endometrial cancer}

\section{RISK OF ENDOMETRIAL CANCER AMONG WOMEN WITH BENIGN OVARIAN TUMORS - A DANISH NATIONWIDE COHORT STUDY}

${ }^{1}$ Kristian Reinholdt, ${ }^{2}$ Susanne Krüger Kjær, ${ }^{1}$ Sonia Guleria, ${ }^{3}$ Kirsten Frederiksen, 'Lene Mellemkjær, 'Christian Munk, 'Allan Jensen. 'Virus, Lifestyle and Genes, Danish Cancer Society Research Center; ${ }^{2}$ Virus, Lifestyle and Genes, Danish Cancer Society Research Center; Department of Obstetrics and Gynecology, Rigshospitalet, University of Copenhagen; ${ }^{3}$ Statistics and Pharmacoepidemiology, Danish Cancer Society Research Center

\subsection{6/ijgc-2020-ESGO.51}

Introduction/Background The few studies investigating a potential association between benign ovarian tumors and endometrial cancer have been inconclusive. Using data from a large Danish register-based cohort study, we assessed the overall and type-specific risk of endometrial cancer among women with a benign ovarian tumor.

Methodology We identified all Danish women diagnosed with a benign ovarian tumor during 1978-2016 in the Danish National Patient Register ( $\mathrm{n}=149,807$ ). The study population was followed for subsequent development of endometrial cancer by linkage to the Danish Cancer Register and standardized incidence ratios (SIRs) with corresponding 95\% confidence intervals (CIs) were calculated after correction for hysterectomy.

Results Women with benign ovarian tumors had a decreased incidence of endometrial cancer (SIR $=0.74,95 \%$ CI: $0.68-$ 0.81) compared with women in the general Danish female population. Both solid benign ovarian tumors $(\mathrm{SIR}=0.79$, 95\% CI 0.70-0.88) and cystic benign ovarian tumors (SIR = $0.68,95 \%$ CI $0.58-0.78)$ were associated with decreased incidences of endometrial cancer. Likewise, women with benign ovarian tumors had decreased incidences of both type I and type II endometrial cancer. The incidence of endometrial cancer was decreased to virtually the same magnitude irrespective of the age at diagnosis of a benign ovarian tumor and the 